\section{The Surgery of the Biliary Tract}

In contrast to the extraordinary safety of operations on the gall-bladder the surgery of the common bile duct is associated with a much heavier risk to life. To make these cases of jaundice safer for surgery operation should be deferred until the jaundice begins to subside. Blood transfusion is better than intravenous calcium chloride as a means of diminishing or preventing the tendency to haemorrhage, and is especially valuable where the bilirubin curve tends to rise. It is also important to administer large quantities of water and of glucose before and after operation-by the rectum or by intravenous infusion. In the worst cases of jaundice nothing more than drainage should at first be essayed.

\section{Genito-Urinary Surgery}

A candidate for the operation of prostatectomy is a " bad surgical risk" if the operation is performed when the blood urea is over $60 \mathrm{mg}$. per $100 \mathrm{c.cm}$. ; and if there is evidence of moderate renal impairment, even in spite of a normal blood urea estimation. Eric Riches and Douglas Robertson $^{21}$ regard it as dangerous to operate radically if the urea-clearance test of van Slyke for estimation of renal function is below 60 per cent. of normal, no matter how normal may be the blood-urea estimation.

Secondary nephrectomy for any condition is always an anxious operation, and two or three transfusions and infusions may be required to carry the patient through his convalescence from what may be a carnivorous type of surgery.

\section{Cenclusion}

It requires no superlative enthusiasm of mine, no unwarranted optimism, to foresee and foretell that, as the "bad surgical risk" of a decade or two ago is now a " normal or good risk," so will the " despairs " of to-day be the "certainties" of the morrow. The craftsmanship of surgery is not yet finished and perfected as an artfresh techniques will be evolved, fresh avenues of approach will be explored, fresh territories will assuredly be conquered. More accurate and certain knowledge of the causation and cure of disease, and methods and forms of therapy still undreamt of, will in future days cheat and rob Death of those victims that, in this twilight of our surgical wisdom, appear to us now beyond all mortal aid.

\section{REFERENCES}

${ }^{1}$ Rooke, Basil: Practitioner, April, 1923, p. 564.

2 Turner, G. Grey: Lancet, December 9th, 1933, p. 315.

s Idem: Proc. Roy. Soc. Med. (Sect. Surg.), June, 1923, xvi, 8, p. 43.

1 Pringle, J. Hogarth: Brit. Journ. Surg., 1916, p. 283.

5 Gordon-Taylor, G., and Wiles, Philip: In the press.

- Duval, Pierre: Soc. de Chir. de Paris, June, 1928.

${ }^{7}$ Green-Armytage, V. B.: Personal communication.

${ }^{B}$ Gordon-Taylor, Hudson, Whitby, Dodds, and Warner: Brit. Journ. Surg., 1929.

- Tyrrell-Gray, H.: Personal communication.

${ }^{10}$ Gabriel, W. B.: Proc. Roy. Soc. Med., May, 1931, xxiv, 5, p. 102.

${ }^{11}$ Bankoff, George: Brittsh Medical Journal, 1934, i, 189

${ }^{12}$ Greene, C. Walters, et al.: Ann. of Surg., December, 1933.

13 Gordon-Taylor, G., Abrahams, A., Bennett, T. Izod, Morley, J., et al.: Lancet, 1934, i, 572.

14 Finsterer, Hans: British Medical Journal, 1932, ii, 399.

15 Wright, Garnett: Association of Surgeons meeting, Birmingham, May, 1934.

${ }^{16}$ Bastianelli, Raffaele: Rep. Internat. Confer. Cancer, 1928, p. 352.

${ }_{17}$ Finsterer, Hans: Ibid., p. 344.

${ }^{18}$ Kelly, R. E.: Proc. Roy. Soc. Med., September, 1929, xxii, 1, 1514.

${ }^{19}$ Sistrunk, W. E.: Collected Papers of the Mayo Clinic, 1928.

20 Turner, G. Grey: Annual Oration, Medical Society of London. Trans. Med. Soc. Lond., 1928-9, lii, 301.

${ }^{21}$ Riches, Eric, and Robertson, Douglas: Personal communication.

\section{RETROVERSION OF THE UTERUS* \\ BY}

S. GORDON LUKER, M.D.CAMB., F.C.O.G. BOURNEMOUTH

The usual position of the uterus is one of anteflexion and anteversion in the middle of the pelvis, the axis of the uterus being very nearly horizontal. When the exact opposite of this occurs the uterus is retroverted and retroflexed. In between these two extremes any position of the uterus may be found. It is usual to describe a uterus as retroverted when the cervix is pointing downwards or forwards and the axis of the body of the uterus is directed backwards. The retroverted uterus is often retroflexed as well.

The normal position of the uterus is maintained by the structures which are attached round the supravaginal portion of the cervix. These are the pelvic fascia, the bases of the broad ligaments, and the utero-sacral ligaments.

There is a wide divergence of opinion as to the symptoms which result from retroversion of the uterus and the treatment required. Some confusion has arisen from grouping all cases together and not separating those of congenital origin from those acquired after pregnancy and labour. It is my intention, therefore, to make as clear as possible the different groups of cases.

\section{Classification}

1. The group of those women who are single and have congenital retroversion. It is a fact that many of them are unconscious of the condition and have no symptoms; these, of course, require no treatment. There are others, however, in whom symptoms begin to appear as they get older. This may be due to increase in degree of the condition, with increasing retroflexion, giving rise to venous congestion and symptoms of backache, increasing dysmenorrhoea, pelvic discomfort, or pain for which no other cause can be found.

2. The group of married women who have a congenital retroversion which gives rise to symptoms, on or after marriage, of which the commonest is dyspareunia; or they may seek advice for sterility; or, if they become pregnant, miscarriage may result. These patients require investigation and treatment.

3 . The group of those who are found to have retroversion acquired after miscarriage or labour. This is a large and important group, as is shown by the record of two hundred consecutive confinements. Post-natal examination one month after delivery showed that of the primiparae 13 per cent. had retroversion; of the multiparae 3.5 per cent. had retroversion. These patients had received the advantage of skilled nursing and postural preventive treatment, so that the percentages are probably higher among the greater number of women attended in their own homes. These require treatment in order to prevent subinvolution after labour; the position of the uterus must be corrected in order to prevent the development of symptoms of pelvic discomfort, backache, congestive dysmenorrhoea, leucorrhoea, and dyspareunia. Further, if a retroverted uterus is left uncorrected after labour it will be a factor leading to future prolapse.

The pelvic discomfort is generally of a dull aching nature, aggravated by activity and fatigue. It may have a bearing-down character or may give rise to a sensation of fullness or pressure on the rectum. The backache is generally dull and constant, increased by fatigue and relieved by rest. It is generally felt posteriorly over the sacrum, but may radiate towards the iliac fossae or the thighs.

* Read in the Section of Obstetrics and Gynaecology at the Annua Meeting of the British Medical Association, Bournemouth, 1934. 


\section{Treatment of Congenital Cases}

In the case of single women many require no treatment and should be left alone. In young married women complaining of dyspareunia or sterility or miscarriage the condition may be treated by manipulation and a pessary, or by operation. It is advisable to give a good trial to medical treatment before resorting to a surgical operation. When treatment is indicated for the above symptoms it is often found that correction of the misplacement cannot be carried out as the manipulations cause pain, and it is difficult or impossible to correct the misplacement. In such cases it is. advisable to carry out an examination under an anaesthetic.

The patient is anaesthetized, a thorough pelvic examination is made, and the body of the uterus is pushed forward either by the fingers alone or with the aid of a vulsellum forceps on the cervix. In difficult cases a uterine sound may be required to assist the reposition of the uterus. Having put the uterus into a satisfactory position, a Hodge pessary is inserted. This is left in for two or three months, and it is then removed and the position of the uterus after removal ascertained. If the uterus remains in good position after this treatment nothing further need be done. If, however, the uterus reverts to its old position after removal of the pessary, the question will arise whether to replace the pessary or to perform an operation. In order to arrive at a decision all the factors in the case must be carefully considered. If the position of the uterus cannot be corrected under an anaesthetic the indications for operative treatment appear greater, but generally speaking the indications for operation in single women do not often arise. In married women, on the other hand, symptoms are often sufficiently important to require treatment by pessary or operation.

\section{Treatment of Asquired Retroversion}

Seeing that retroversion is most often the result of pregnancy and labour, it is very important to adopt all precautionary measures which may prevent the occurrence of this displacement. The most important of these precautionary measures consist in promoting the free drainage and anteversion of the uterus during the puerperium. The puerperal woman should assume a prone position for at least two hours in the day. The bladder should be emptied at regular intervals, and the abdominal binder should not be sufficiently tight to press the fundus of the uterus back. Adequate rest in bed is essential, but it is difficult, for economic reasons, to arrange for hospital patients to stay in bed for more than twelve days. At the end of a month a post-natal examinat in should be made, and the position of the uterus deterinined. If the uterus is retroyerted the position must be corrected by manipulation, and a Hodge pessary inserted for two months. At the same time the patient must be instructed to do all she can to promote involution of the uterus and pelvic floor by suitable exercises.

General lines of treatment fall under three headings: exercises; pessaries, and operation.

Exercises are most useful in helping to restore tone to the uterine supports after labour during the puerperium. They are helpful at a later date, but are of more value in restoring loss of tone than in repairing injury to the tissues. Further, their scope is limited, because it is difficult to give instruction in exercises designed to strengthen the levator ani muscle and the uterine supports, and it is even more difficult to get women to carry out these exercises except under expert instruction.

Treatment with Pessaries. - The vulcanite Hodge pessary is the instrument most suitable for temporary treatment of retroversion, and is far superior to the rubber ring for this purpose. Indications for its use are: (a) In mobile puerperal retroversion after correction of the position of the uterus. The pessary should be left in two months, during which time the pelvic floor and uterine supports regain their normal tone. On removing the pessary the uterus should remain in a position of anteversion. (b) In non-puerperal mobile retroversion-that is to say, when the retroversion is discovered at some interval after the last confinement. Here the pessary treatment is not likely to effect a permanent cure, but will relieve symptoms and arrest the progress of the displacement. When used in these circumstances the pessary will act as a valuable diagnostic agent, for if symptoms are cured by its introduction it shows that they were due to the backward displacement of the uterus. This will be of value in deciding whether operative treatment should be undertaken. (c) Pessaries are useful after correction of a backward displacement in early pregnancy; the instrument will prevent the recurrence of the retroversion. The main indications for pessary treatment are as a therapeutic test and as a temporary measure in puerperal retroversion. When, however, pessary treatment cannot effect a permanent cure, and relapse occurs after removal, the time has come to consider the advantages of operative treatment.

\section{Operative Treatment of Retroversion}

The indications for operative treatment for backward displacement of the uterus are in some cases quite clear when symptoms are severe or cause the patient considerable discomfort, or cannot be alleviated by pessaries or other means. This may occur when the uterus is fixed and the cvaries are prolapsed and tender ; when the retroversion is associated with pelvic inflammation; when there is dyspareunia; when there is sterility; and when there is abortion. A decision, on the other hand, may be difficult when the symptoms are not severe, and when the retroversion is uncomplicated.

The question of operation is influenced by the age of the patient less than are many operations, for the reason that after operation pregnancy and labour may supervene without any risk of complication or recurrence. Before deciding to operate it is most important to take a very thorough history, make a very complete examination, and to have no doubt that the symptoms referred to are not caused by anything apart from the displacement.

In order to ascertain the opinion of others as to the indications for operative treatment in uncomplicated retroversion a form of inquiry was sent to the senior gynaccologists at the London teaching schools. The result of this inquiry shows that operative treatment is not common. When it is done the commonest indications are sterility and dyspareunia. The present position seems to be against operative treatment in uncomplicated cases. This attitude should, I think, be modified, since there are many patients who suffer severe symptoms from an uncomplicated retroversion who could be cured by operative treatment.

The indications for operation are, in my opinion, as follows :

(a) When the retroversion is fixed or is complicated by adhesions or by chronic pelvic inflammation.

(b) When the retroversion is giving rise to symptoms, which are increasing, of backache, pelvic pain, menorrhagia, dysmenorrhoea, or pain and difficulty with micturition or defaecation.

(c) When the retroversion, though mobile and simple, causes symptoms which disappear after correction of the displacement and insertion of a pessary - for example, dyspareunia.

(d) When retroversion is causing sterility.

(e) When retroversion is causing miscarriage.

In uncomplicated cases it is important to use a pessary as a therapeutic test before deciding in favour of operation; if the pessary relieves the symptoms, permanent cure by operation may be looked for with confidence. 


\section{Nature of the Operation}

The operation of choice is that generally described as the modified Gilliam operation, after the name of the surgeon who first described this operation in the year 1900. The principal points in the operation are that a loop of the round ligament on each side is brought out under the peritoneum and attached to the anterior rectus sheath on each side. Details of the operation are as follows.

The abdomen is opened by a paramedian incision. A stitch of No. 3 chromicized catgut is tied about the round ligament on each side an inch from the cornu of the uterus. The anterior rectus sheath is then cleared, and a small puncture is made towards the outer part at the level of the fundus of the uterus. A special curved forceps is then passed outwards behind the peritoneum till the region of the internal abdominal ring is reached. The forceps then turns inwards in the base of the broad ligament till its point reaches the ligature on the round ligament. The peritoneum is then punctured, the round ligament suture grasped with the forceps and withdrawn along the track made by the forceps through the rectus sheath. The loop of round ligament is then made fast to the rectus sheath and the abdomen closed. The suture material used is No. 3 chromicized catgut, as this has been found satisfactory and no recurrence has occurred.

This operation has stood the test of time and has given complete satisfaction. It has therefore seemed unnecessary to change the technique of the operation or the suture material. In order to confirm these points, letters were written to patients operated upon from two to fourteen years ago, inquiring as to the result of the operation. The replies were illuminating and highly satisfactory.

\section{Modifications of Operation}

In order to test my own results $I$ sought the opinion of the senior gynaecologists of the London teaching schools as to the nature of operation employed. All appear to employ a modified Gilliam operation, and find it eminently satisfactory. Many seem to prefer the use of silk, and some think it essential. Many, however, give up puncturing the rectus sheath, and draw the loop of round ligament up between the outer surface of the rectus muscle and the under surface of the rectus sheath. One operator pleats up the round ligament before drawing it through to the under surface of the rectus sheath, to which it is firmly attached by silk, thus approaching the technique of a fixation operation. Another operator pleats up the round ligaments and attaches them to the cornu of the uterus; while a third combines the ventral suspension of the uterus with shortening of the utero-sacral ligaments. Except for these three modifications, all do the modified Gilliam operation, and are satisfied with the results. The majority use silk sutures, but I have found chromicized catgut satisfactory, and think that silk introduces a possible element of sepsis.

\section{Conclusions}

Retroversion of the uterus is responsible for a considerable amount of ill-health in women. It may be prevented or cured during the puerperium by suitable exercises and treatment.

Congenital retroversion in single women often causes no symptoms and requires no treatment.

Congenital retroversion in married women does cause symptorns.

Retroversion acquired after labour or miscarriage is common, and the importance of preventive treatment is stressed.

Acquired retroversion often gives rise to symptoms, and requires correction by pessary or operation.

\section{HAEMOPTYSIS : A NOTE ON PATHOLOGY} AND TREATMENT

BY

\author{
H. V. MORLOCK, M.C., M.D., M.R.C.P. \\ AND
}

A. J. SCOTT PINCHIN, M.D., F.R.C.P. PHYSICIANS TO CITY OF LONDON HOSPITAL FOR DISEASES OF HEART AND LUNGS

Haemorrhage from organs such as the lungs and intestines, which are not accessible to the ordinary surgical methods of haemostasis, present a problem which is peculiar to itself ; for not only does the practitioner feel more or less helpless, as he is unable to reach the bleeding point, but the psychological effect on the patient of bringing up large quantities of blood-whether from lungs or stomach - together with the knowledge that the lesion is not accessible, leads to a distress of mind which reacts unfavourably on the natural processes which should physiologically help to stay the haemorrhage.

The usual treatment recommended for haemoptys:s is along the general lines suggested for the arrest of bleeding when not accessible to direct methods of haemostasis; these general methods have two objects in view, which are as follows. In the first place, to secure a local effect by the administration of drugs or other preparations which have the reputation for promoting clotting at the source of the haemorrhage by increasing the coagulability of the blood ; secondly, to endeavour to rest the organ from which the bleeding is taking place, and by sedatives to quiet the body, ease the mind, and so cause a reduction in the rate of flow of the blood.

Preparations used in the first group, such as calcium, haemostatic sera, horse sera, etc., whilst possibly of some use are somewhat unreliable in their action, and, in consequence, though used as a routine, they are regarded as ràther accessory, and there is a natural tendency to place most reliance on those methods which will promote mental quiet and physical rest to the organ from which the haemorrhage is proceeding, and thus give the bleeding vessel the opportunity of forming a clot sufficient to stay the haemorrhage.

\section{Pathology and Mechanics of Haemoptysis}

Should these methods be successful in stopping the haemorrhage the arrest is only slowly produced, with the result that some bleeding will continue to take place for varying periods, according to the size of the vessel eroded, while in addition any movement or disturbance of the patient may cause the haemorrhage to recommence, and as a result a considerable amount of blood will be found in the lumen of the organ concerned.

If the bleeding is intestinal the blood in the lumen will be drained into the intestinal tract, but in a haemoptysis the blood remains in the bronchus and can only be evacuated therefrom by the patient coughing; if, however, the patient has been forbidden to cough, and has been heavily morphinized, the cough reflex will be lost, and the blood will remain in the bronchus. It is well recognized that even during natural sleep secretions from the upper respiratory tract may be aspirated into the lower bronchi ; further, it is admitted that, when unconsciousness is increased by morphine or basal anaesthetics, aspirated secretions may cause a pulmonary collapse. In the morphinized patient, with blood or blood clot in the bronchial tree, the chances of mechanical pathological changes taking place are very considerable.

If the quantity of blood in the bronchus is small, aspiration into the smaller bronchi and bronchioles will take place; further, if the patient gives a half-stifled ineffective cough, the blood will be evacuated from the 